\title{
Rescue under ongoing CPR from an upper floor: evaluation of three different evacuation routes and mechanical and manual chest compressions: a manikin trial
}

\author{
Hendrik Drinhaus ${ }^{1,2^{*}}$ (D), Sebastian Nüsgen ${ }^{2}$, Niels Adams ${ }^{1,2}$, Wolfgang A. Wetsch ${ }^{1}$ and Thorsten Annecke
}

\begin{abstract}
Background: If transport under ongoing cardiopulmonary resuscitation (CPR) from an upper floor is indicated, the ideal CPR-method and evacuation route is unknown hitherto. We aimed to elaborate a strategy for evacuation of patients under ongoing CPR from an upper floor, comparing three different evacuation routes and manual and mechanical chest compressions.

Methods: A CPR-training manikin recording CPR-quality was placed on the fifth floor and was evacuated to an ambulance via lift, turntable ladder, or staircase. Chest compressions were performed manually or with a mechanical CPR-device. Efficiency endpoints were compression depth and frequency, sufficiency of chest release, compared with European Resuscitation Council (ERC) Guidelines, and duration of the evacuation. Adverse outcomes were disconnection/dislocation of devices and hazards/accidents to the personnel.

Results: For all evacuation routes, compression depth and frequency were significantly more compliant with ERCguidelines under mechanical CPR. Manual CPR was associated with considerable deviations from correct compression depth and frequency. Chest release only slightly differed between groups. Evacuation via lift under mechanical CPR was fastest and evacuation via turntable ladder under manual CPR was slowest. No device disconnections or accidents occurred, but hazard to personnel was perceived during evacuation via ladder under manual CPR.

Conclusions: In this study, a mechanical CPR-device proved to deliver better CPR-quality during evacuation from an upper floor. If a lift accessible with a stretcher is available, this route should be preferred, regardless of manual or mechanical CPR. Turntable ladders can only be meaningfully used with mechanical CPR, otherwise CPR-quality is poor and hazard to the personnel is increased. Not all evacuation routes may be useable in a specific real-life scenario.

Trial registration: German Clinical Trials Registry, www.drks.de, registration number DRKS00012885, registration date 17.08.2017.

Keywords: Out-of-hospital cardiac arrest, Cardiopulmonary resuscitation, Emergency medical services, Transport under ongoing cardiopulmonary resuscitation, Fire service, Mechanical chest compressions
\end{abstract}

\footnotetext{
* Correspondence: hendrik.drinhaus@uk-koeln.de

${ }^{1}$ Department of Anaesthesiology and Intensive Care Medicine, University of

Cologne, Faculty of Medicine and University Hospital of Cologne, Kerpener

Str. 62, 50937 Cologne, Germany

${ }^{2}$ Fire Brigade, Brühl, North Rhine-Westphalia, Germany
}

(c) The Author(s). 2020 Open Access This article is distributed under the terms of the Creative Commons Attribution 4.0 International License (http://creativecommons.org/licenses/by/4.0/), which permits unrestricted use, distribution, and reproduction in any medium, provided you give appropriate credit to the original author(s) and the source, provide a link to the Creative Commons license, and indicate if changes were made. The Creative Commons Public Domain Dedication waiver (http://creativecommons.org/publicdomain/zero/1.0/) applies to the data made available in this article, unless otherwise stated. 


\section{Background}

Out-of-hospital cardiac arrest (OHCA) is usually treated by performing cardiopulmonary resuscitation $(\mathrm{CPR})$ on scene until return of spontaneous circulation (ROSC) is achieved or until a decision is taken to terminate CPR in physician-staffed emergency medical services (EMS). Transport to a hospital under ongoing CPR is rare in such systems. In selected cases, however, transporting the patient still in cardiac arrest to a hospital for causal treatment of a potentially reversible cause of cardiac arrest is warranted $[1,2]$. Some EMS-systems prefer establishing extracorporeal cardiopulmonary resuscitation (eCPR) by implanting a veno-arterial extracorporeal membrane oxygenation (ECMO) on scene [3], whereas other concepts recommend transporting the patient to the hospital under ongoing CPR and establishing ECMO in-hospital [1]. Performing high-quality CPR during transport (consisting of evacuation from the scene to the ambulance, drive to the hospital, and transfer to treatment facility therein) can be challenging. Several aspects have to be considered to decide on the optimal transport modalities, including possibility and potential quality of chest compressions, length and speed of transport, required alterations of patients' position, required personnel and material, as well as hazards to EMS-staff. In order to deliver high-quality chest compressions and to reduce risks of injury to the personnel during patient transport, mechanical CPR devices can potentially be helpful, even though their use has not proven to be superior to manual $\mathrm{CPR}$ and is therefore not recommended for standard CPR in a static situation [4-8]. Additionally, there is a debate about the possibly increased risk of patient injury related to mechanical chest compression devices. To evacuate patients under ongoing CPR from an upper floor, technical and personal assistance from the fire service is usually helpful and close cooperation between EMS and the fire service is crucial. In this study, we sought to establish the optimal strategy for evacuation of a simulated patient under ongoing CPR from the fifth floor. To this end, evacuation via lift, turntable ladder, or staircase was tested, using either manual or mechanical CPR.

\section{Methods}

\section{Registration and ethical approval}

The study was registered in the German Clinical Trials Registry (Deutsches Register für Klinische Studien, www.drks.de, registration number 00012885) and ethical approval was obtained from the institutional ethics committee of the Medical Faculty of the University of Cologne (approval number 17-301).

\section{Study participants}

This study was integrated into the compulsory annual training of paramedics at the fire brigade of the city of Brühl, North Rhine-Westphalia, Germany. The participants were 40 (33 male, 7 female) paramedics of different qualifications (19 Rettungssanitäter [lower qualification, similar to EMT in many US states], 17 Rettungsassistenten [traditional higher qualification, similar to AEMT in many US states] and 4 Notfallsanitäter [new higher qualification, similar to AEMT in many US states] in the German system), between 18 and 60 years of age. They had been informed about the planned scientific evaluation of the training and agreed to participate. No data related to individual participants was collected during the study.

\section{Experimental setup}

Due to the rareness of the scenario in real life and lack of comparability between potential incident scenes, we chose a manikin study design. A CPR-training manikin (Resusci Anne, Laerdal, Stavanger, Norway) was mounted on a spine-board, connected to a defibrillator (Corpuls C3, GS Elektromedizinische Geräte Stemple, Kaufering, Germany) and a respirator (Medumat Standard, Weinmann, Hamburg, Germany). Additional weights were attached to generate a more realistic total weight of $75 \mathrm{~kg}$. Participants were divided into groups of eight (representing two ambulance staff, two emergency physician dispatch car staff, and four fire engine staff). We simulated different evacuation routes and CPR-techniques in a rotating manner, i. e. all participants performed all scenarios during the training day.

To avoid bias due to exhaustion, groups took turns in participation and their roles during resuscitation. Distribution between participants of higher and lower training grades was balanced. Blinding of the participants was not possible. Due to obvious differences in the results observed between manual and mechanical CPR, blinding of data analysts would not have been reasonably possible, either. Each experiment was performed three to five times. The intended number of executions of each scenario was five, there was one dropout in the lift/manual group due to unavailability of the lift, two in the ladder/mechanical group (one due to unavailability of the ladder, one due to a failure of data registration in the SimPad), and one in the stairs/mechanical group due to a failure of data registration in the SimPad (Fig. 1).

In the groups allocated to mechanical CPR, a pistonbased CPR-device (Corpuls CPR, GS Elektromedizinische Geräte Stemple) of the EMS of the fire brigade of Brühl was applied. The so-equipped manikin was placed on the floor of an apartment in the fifth floor (UK numbering) of a retirement home. As the aim of our study was to investigate duration and quality of CPR during the evacuation from the building, we measured these 


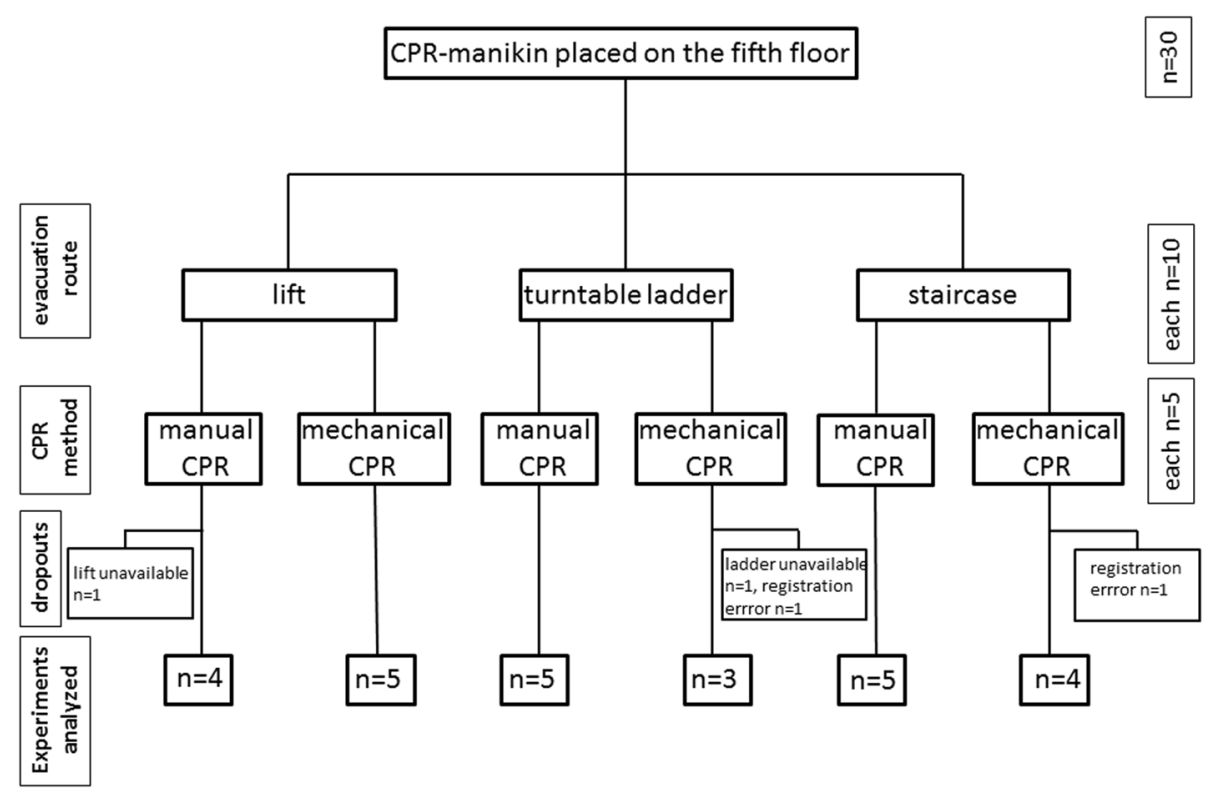

Fig. 1 Flowchart of the study setup

parameters from the time of lifting the spine board until arrival at an ambulance parked in front of the building. CPR-events before the start of evacuation, such as endotracheal intubation or attachment of the CPRdevice, were not part of the study.

For evacuation via lift, the manikin was lifted on an ambulance stretcher (Stryker M-1, Stryker, Kalamazoo (MI), USA) and transported via a stretcher accessible lift. Participants were free to perform CPR either while walking beside the stretcher or squatting on it. For evacuation via staircase, the spine-board was carried through a large staircase under ongoing CPR. For evacuation via turntable ladder, the upper part of the stretcher was mounted on the stretcher swivel support of the rescue cage of a turntable ladder (DLK 23/12, Magirus, Ulm, Germany) (Additional file 1: photography 1). One firefighter wearing a security harness stayed in the rescue cage and performed chest compressions in the manual CPR group.

\section{Endpoints of the study}

CPR quality was directly measured using a resuscitation training manikin with digital quantification and recording of all CPR measures. Efficiency endpoints were compression depth, frequency of compressions, residual compression of the chest between each compression as a measure of leaning on the chest, and duration of the evacuation. Adverse outomes were disconnection or gross dislocation of the intravenous line, respirator, defibrillator or CPR-device as well as accidents or perceived dangers (subjective measure by the participants and an additional observer) to the personnel during the evacuation.

\section{Data collection}

Duration of evacuation and data on CPR-quality were recorded by a tablet computer (SimPad, Laerdal) connected to the CPR-manikin. Data was extracted from the software provided by the manufacturer (SimView, Laerdal) and transferred to Microsoft Excel for further analysis. Compression depth was measured by the device, registered by the software of the manikin and displayed in $1 \mathrm{~mm}$ intervals. Accuracy, according to the manufacturer, is $\pm 15 \%$ or $3 \mathrm{~mm}$. Compression frequency was calculated from the time elapsed between two compressions, thus representing a beat-to-beat frequency analysis, not the mean value within a longer (e. g. one minute) time frame. Statistical analysis and graphical layout were performed with Graph Pad Prism 7 (GraphPad Software, San Diego (CA), USA). For comparisons between two groups, the Mann Whitney test was used. For comparison between two independent variables and their interaction, two-way ANOVA was used. A $p$-value of $<0.05$ was considered statistically significant.

\section{Results}

\section{Compression depth}

The compression depth recommended by the guidelines of the ERC is 50 to $60 \mathrm{~mm}$. In our study, compression depth was significantly lower in all manual groups compared with the respective mechanical groups (Fig. 2a, Table 1). Among the manual compression groups, the 

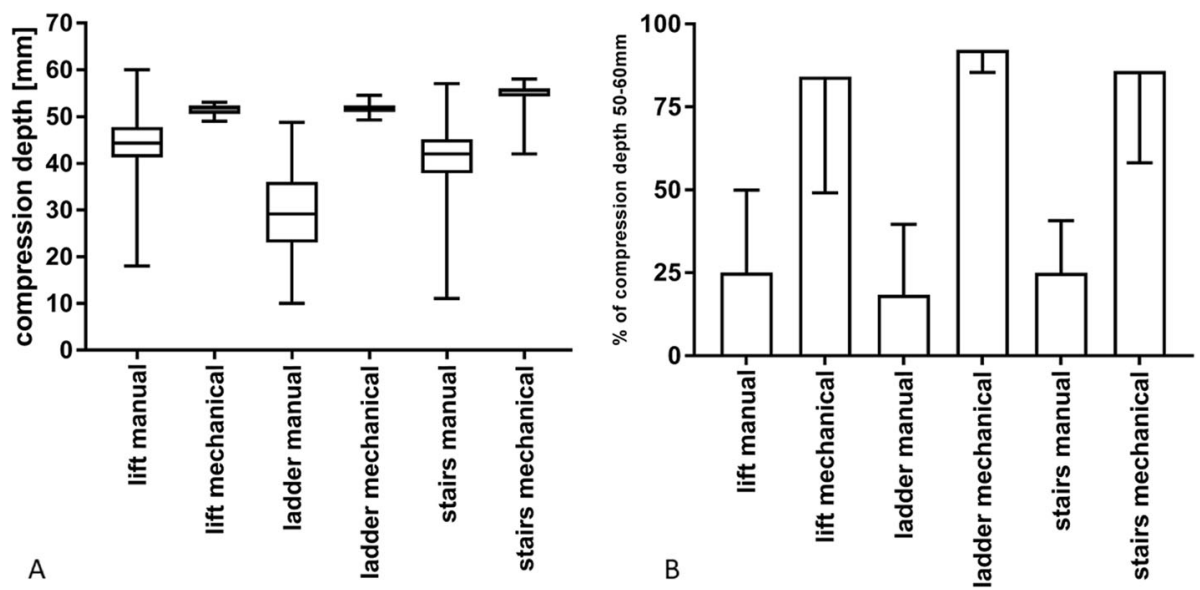

Fig. 2 a: Compression depth in millimetres. Box whisker plot with minimum, maximum, 25th and 75th percentile and median. b: Percentage of guideline-compliant compression depth 50-60 mm. Means and standard deviation (SD)

lowest median compression depth was observed during evacuation via ladder. Especially during the phase of actual movement of the ladder from the fifth floor to the ground, compression depths were low (Additional file 2: Figure S1). The percentage of compression with a depth of 50 to $60 \mathrm{~mm}$, as recommended by current guidelines, was higher with mechanical CPR in all groups (Fig. 2b, Table 1) than with manual CPR, which yielded a lower percentage of correct compression depth. Additionally, in the mechanical groups those compressions not in the target range were almost all very close to its lower end of $50 \mathrm{~mm}$ (Additional file 2: Figure S1). Two-way ANOVA showed a statistically significant influence of CPR-method (manual versus mechanical, $p<0.001$ ) but not evacuation route $(p=0.55)$ on compression depth, and no interaction between CPR-method and evacuation route $(p=0.64)$.

\section{Frequency of compressions}

The compression frequency recommended by the guidelines of the ERC is 100 to 120 compressions per minute. In our study, wide variations of compression frequency were measured in all manual groups whereas a frequency close to the prescribed $100 /$ min was maintained in all mechanical groups (Fig. 3a, Table 2). The percentage of compressions of a frequency of 100 to 120 per minute, as recommended by current guidelines, was very high in the mechanical groups, mediocre in the lift/manual and ladder/manual, and lowest in the stairs/manual group (Fig. 3b, Table 2). Particularly long hands-offtimes were observed in the manual ladder evacuation group during loading the spine-board onto the ladder basket. Here, repetitive interruptions of up to five seconds each occurred (Additional file 2: Figure S1). Twoway ANOVA showed a statistically significant influence of CPR-method $(p<0.001)$ but not evacuation route $(p=0.27)$ on compression frequency, and no interaction between CPR-method and evacuation route $(p=0.28)$.

\section{Residual compression between chest compressions / insufficient chest release}

The guidelines of the ERC recommend total release of the chest between compressions. In our study,

Table 1 Compression depth

\begin{tabular}{|c|c|c|c|}
\hline Evacuation route & Manual compression & Mechanical compression & $p$-value \\
\hline \multicolumn{4}{|l|}{ lift } \\
\hline Compression depth (mean \pm SD) $[\mathrm{mm}]$ & $44 \pm 5$ & $51 \pm 1$ & $<0.001$ \\
\hline$\%$ guideline-compliant depth (mean \pm SD) & $25 \pm 25$ & $84 \pm 35$ & 0.06 \\
\hline \multicolumn{4}{|l|}{ ladder } \\
\hline Compression depth (mean \pm SD) $[\mathrm{mm}]$ & $30 \pm 8$ & $52 \pm 1$ & $<0.001$ \\
\hline$\%$ guideline-compliant depth (mean \pm SD) & $18 \pm 21$ & $92 \pm 7$ & 0.04 \\
\hline \multicolumn{4}{|l|}{ stairs } \\
\hline Compression depth (mean \pm SD) $[\mathrm{mm}]$ & $41 \pm 7$ & $55 \pm 2$ & $<0.001$ \\
\hline$\%$ guideline-compliant depth (mean \pm SD) & $25 \pm 16$ & $86 \pm 28$ & 0.02 \\
\hline
\end{tabular}



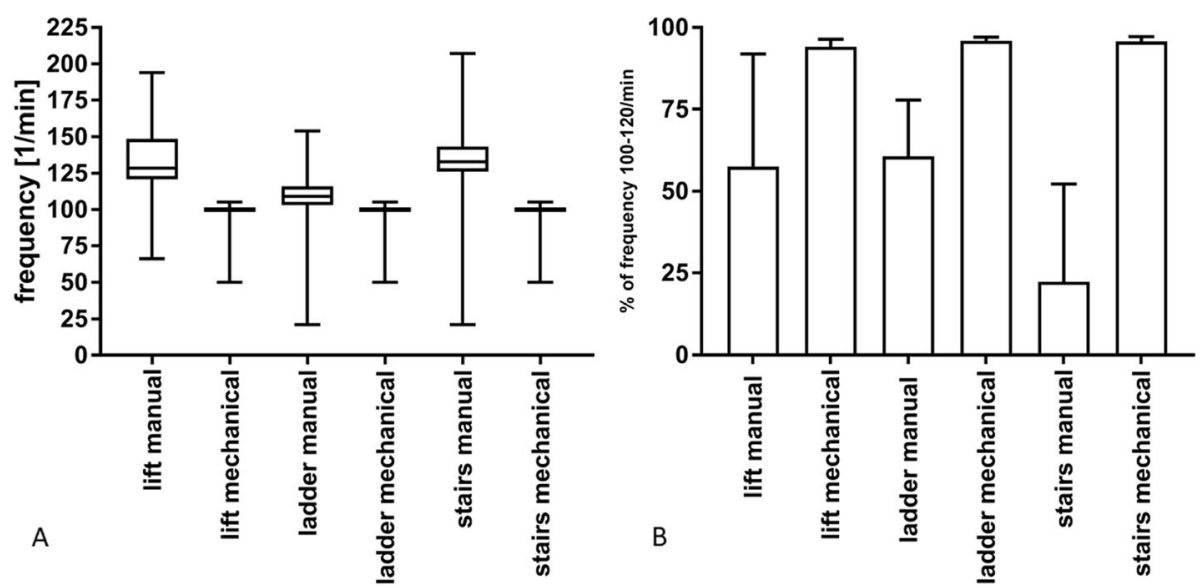

Fig. 3 a: Compression frequency in 1/min. Box whisker plot with minimum, maximum, 25th and 75 th percentile and median. b: Percentage of guideline-compliant compression frequency 100-120/min. Means and standard deviation (SD)

insufficient chest release between compressions varied between groups with a pattern less clear than for compression depth or frequency. For lift and ladder, residual compression of the chest as a measure of insufficient release was more prominent in the manual groups, whereas during evacuation via the staircase, there was more insufficient chest release in the mechanical group (Fig. 4, Table 3). Two-way ANOVA showed no statistically significant influence of CPR-method $(p=0.9)$ or evacuation route $(p=0.33)$ on residual compression, and no interaction between CPR-method and evacuation route $(p=0.09)$.

\section{Duration}

Mean duration of transport reached between $152 \mathrm{~s}$ in the lift/mechanical group and $306 \mathrm{~s}$ in the ladder/manual group. For each evacuation route, evacuation with mechanical CPR was numerically shorter but these differences did not reach statistical significance in the separate analyses of each evacuation route ( $p=0.127-0.257)$ (Fig. 5).
In the overall analysis of all experiments with two-way ANOVA a statistically significant influence was found for CPR-method $(p=0.04)$, but not for evacuation route $(p=0.23)$ or for interaction between both $(p=0.76)$.

\section{Adverse outcomes}

No disconnections of any device occurred; gross dislocations of the CPR-device were not observed while slight dislocations could not be ruled out by the experimental setup. There were no accidents to the personnel or bystanders. Perceived danger as reported by the participants and the observer to the firefighters performing manual CPR in the turntable ladder basket was high, especially when mounting on the metal bars of the basket to increase efficiency of chest compressions (Additional file 3: photography 2).

\section{Discussion}

We have compared three different evacuation routes and two different CPR-techniques during evacuation under ongoing CPR from a flat in the fifth floor. For all

Table 2 Compression frequency

\begin{tabular}{|c|c|c|c|}
\hline Evacuation route & Manual compression & Mechanical compression & $p$-value \\
\hline \multicolumn{4}{|l|}{ lift } \\
\hline Compression frequency (mean $\pm \mathrm{SD}$ ) [mm] & $133 \pm 17$ & $99 \pm 4$ & $<0.001$ \\
\hline$\%$ guideline-compliant frequency (mean \pm SD) & $58 \pm 34$ & $94 \pm 2$ & 0.02 \\
\hline \multicolumn{4}{|l|}{ ladder } \\
\hline Compression frequency (mean $\pm \mathrm{SD}$ ) [mm] & $108 \pm 14$ & $100 \pm 4$ & $<0.001$ \\
\hline$\%$ guideline-compliant frequency (mean \pm SD) & $61 \pm 17$ & $96 \pm 1$ & 0.04 \\
\hline \multicolumn{4}{|l|}{ stairs } \\
\hline Compression frequency (mean $\pm \mathrm{SD}$ ) $[\mathrm{mm}]$ & $135 \pm 22$ & $100 \pm 4$ & $<0.001$ \\
\hline$\%$ guideline-compliant frequency (mean \pm SD) & $22 \pm 30$ & $96 \pm 2$ & 0.02 \\
\hline
\end{tabular}


Table 3 Residual compression

\begin{tabular}{|c|c|c|c|}
\hline Evacuation route & Manual compression & Mechanical compression & $p$-value \\
\hline \multicolumn{4}{|l|}{ lift } \\
\hline Residual compression (mean \pm SD) [mm] & $6 \pm 3$ & $3 \pm 1$ & $<0.001$ \\
\hline \multicolumn{4}{|l|}{ ladder } \\
\hline Residual compression (mean \pm SD) [mm] & $3 \pm 3$ & $1 \pm 1$ & $<0.001$ \\
\hline \multicolumn{4}{|l|}{ stairs } \\
\hline Residual compression (mean $\pm \mathrm{SD}$ ) $[\mathrm{mm}]$ & $2 \pm 2$ & $4 \pm 1$ & $<0.001$ \\
\hline
\end{tabular}

Means, standard deviation, and $p$-value of residual compression for each evacuation route and CPR-method

evacuation routes, mechanical chest compressions were found to provide superior CPR-quality in terms of compression depth and frequency. Using a CPR-device, no significant differences in CPR-quality could be detected for evacuation via lift (accessible with a stretcher), turntable ladder, or staircase. For manual chest compressions, particularly poor CPR-quality as well as hazards to the staff were observed during evacuation via turntable ladder. The best results for manual CPR were obtained during evacuation via lift.

Poor manual CPR-quality while moving patients is in accordance with prior publications that studied CPRquality during other phases of the overall patient transport, e.g. in an ambulance or a helicopter [9-11]. While human and manikin studies focused on "technical" parameters of CPR-quality, such as compression depth and frequency and hands-off-time, a recent trial in a porcine model analysed haemodynamic parameters during manual or mechanical CPR in a moving ambulance. Mechanical resuscitation with the LUCAS-device resulted in

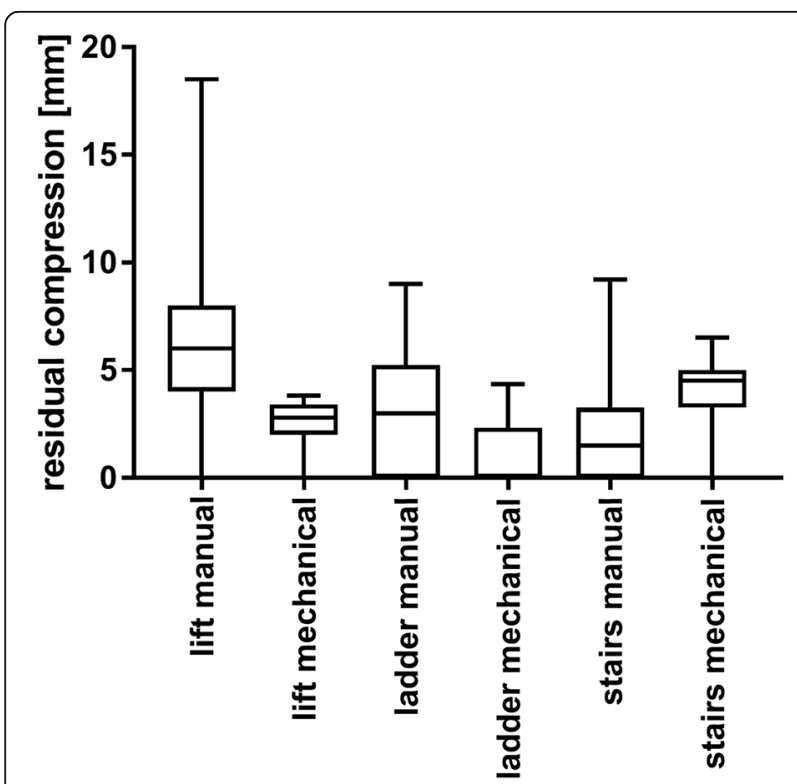

Fig. 4 Residual compression of the chest between compressions in millimetres. Means and standard deviation (SD) higher coronary perfusion pressure, higher end-tidal $\mathrm{CO}_{2}$ and lower lactate levels than manual chest compressions [12].

To the best of our knowledge, the phase of transport looked at here - evacuation from a flat in a upper floor to the ambulance - has not been studied in detail yet.

One manikin trial compared CPR-quality with manual or mechanical (LUCAS-device) CPR during transport from a second floor to the hospital, thus including the phase analysed here. The only evacuation route in that study was the staircase. Mechanical CPR was found to result in a higher percentage of compressions with adequate depth (LUCAS $52 \%$ vs. manual $36 \%, p<0.07$ ) and rate (LUCAS $71 \%$ vs. manual $40 \%, p<0.02$ ) during the overall transport. A separate analysis of the phase of evacuation through the staircase is not available [13].

A patient trial analysed manual and mechanical (AutoPulse-device) CPR while moving the patient on a flexible extrication sheet from the site of collapse into the ambulance. No information concerning the sites of

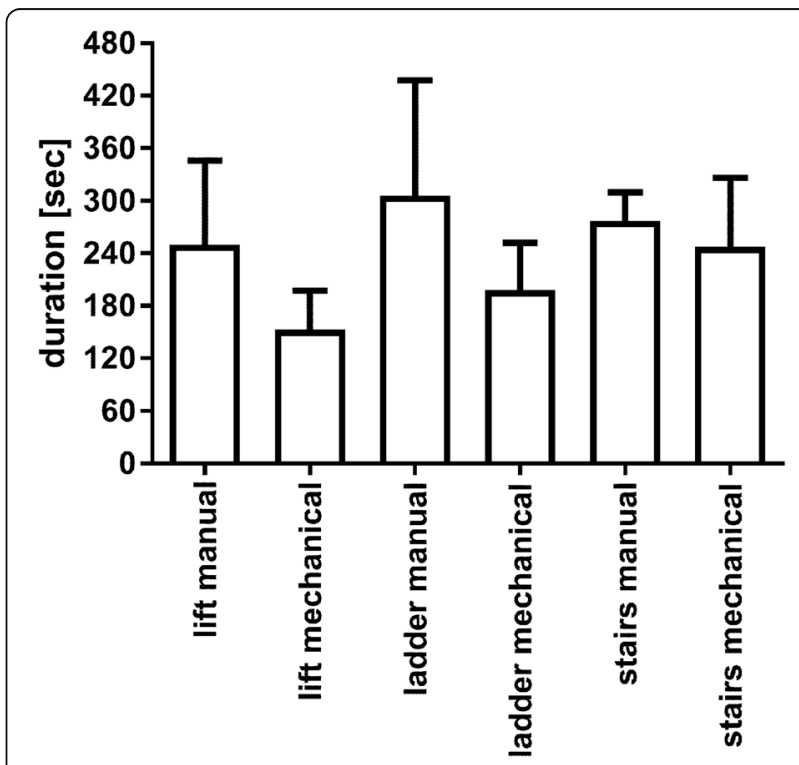

Fig. 5 Duration (in seconds) of transport from the fifth floor to the ambulance in front of the building. Means and standard deviation (SD) 
collapse is available, so the percentage of patients evacuated from a building, as compared with those evacuated from e. g. a nearby pavement, is unknown. The range of 32 to $540 \mathrm{~s}$ for the extrication suggests a diversity of locations. In the manual-CPR-group, chest compressions were interrupted for a median of $270 \mathrm{~s}$ versus $39 \mathrm{~s}$ in the AutoPulse-group [14].

OHCA in upper floors appears to be related to lower survival rates. Besides demographic or sociological factors, this finding is linked to longer EMS response times. Whether difficulties in evacuation from the upper floors, with or without ongoing CPR, may play a role, cannot be judged from the data provided in the according publications $[15,16]$. Our results suggest that in cases of transport under ongoing CPR without a CPR-device, poor CPR-quality has to be expected, possibly contributing to lower survival rates.

From the findings in our study, we suggest using a CPR-device whenever the decision is taken to evacuate a patient under ongoing CPR from an upper floor. If a stretcher accessible lift is available, this route should be preferred, whether CPR be mechanical or manual. Apart from a relatively sufficient CPR-quality for manual CPR, this route avoids tilting of the patient and reduces the need for personnel and material, possibly obviating the need for support from the fire service. If the location of collapse can be accessed with a turntable ladder, this is a suitable route given the availability of a CPR-device. If this is unavailable, CPR-quality during loading and moving of the ladder as well as hazard to the staff is too high to recommend this route. Evacuation via the staircase should be avoided if possible, as it implies tilting of the patient, going along with possible risk of gross dislocation of the CPR-device (which was not observed in our experiments) and strain to personnel (which is required in large numbers). Whether for lower floors than studied here, the practicability of a short staircase transport, compared with bringing a turntable ladder into position, might outweigh these drawbacks, remains undecided from our model of evacuation from the fifth floor.

It remains unclear why chest release between compressions with the Corpuls CPR-device was worse during transport via staircase compared with the other routes. One possible explanation might be slight dislocations of the piston, which is applied with a certain surface pressure at the beginning of treatment, resulting in insufficient release of the chest. Another possible reason might be an oversensitivity of the resuscitation manikin's pressure sensor.

There is a discussion about the potential of CPR devices to cause musculoskeletal or visceral injuries, such as liver or spleen lacerations. The only randomised trial of devicerelated injuries so far did not include the Corpuls CPRdevice used in this study, but the piston-based device analysed in that study, LUCAS, was found to be noninferior to manual CPR in terms of severe injuries [17]. By contrast, in two other recently published large studies, patients resuscitated with the LUCAS-device had more resuscitation-related injuries $[18,19]$, even though this effect lost statistical significance after adjusting for CPRduration in one of the studies [18]. In case of evacuation from an upper floor, we would still suggest using CPRdevices, even if their injury potential were higher, because of the improvement of CPR-quality versus manual CPR, which performs poorly in this situation.

We recognise three main limitations of this study. Firstly, a manikin study obviously does not permit the analysis of clinical endpoints, such as ROSC, survival or neurological outcome, but had to focus on "technical" parameters of CPR-quality. The low number of real cases, however, precluded the possibility to study this research question in real patients. Secondly, the number of repetitions for each experiment was low. Still, we believe that even with a limited number of repetitions our results are clear enough to support our findings and conclusions in a robust manner. Thirdly, we looked at a clearly defined, isolated phase of transports with all means and personnel readily available at the start of the experiment. In real-life-scenarios, time of attachment of the CPR-device, availability and driving time of the fire service or dimensions of lift or staircase are aspects to be considered. Time intervals such as duration from arrival at the scene to first chest compression or to starting transport to the hospital were not part of this study. Furthermore, participants and investigators were not blinded to the chest compression method and extrication route, which might potentially create a bias. However, CPR-quality during the short stationary phase of the experiment appeared comparable to the CPR-quality observed during general CPR-drills of the fire brigade.

\section{Conclusions}

Our manikin study has shown that mechanical CPR is more effective to perform consistent high quality CPR during evacuation under ongoing CPR from an upper floor. Without mechanical CPR, significant deviations from guideline-recommendations were observed, particularly for evacuation via turntable ladder or stairs. If a stretcher accessible lift is available, this evacuation route offers the possibility of good CPR-quality with low personnel and technical effort. During evacuation via turntable ladder, manual CPR goes along with insufficient CPR-quality and hazards to the staff.

\section{Supplementary information}

Supplementary information accompanies this paper at https://doi.org/10. 1186/s13049-020-0709-0. 
Additional file 1. Photograph of the manikin fully equipped being loaded onto the turntable ladder.

Additional file 2. Representative original tracings of compression depth and frequency from the Laerdal Session Viewer software.

Additional file 3. Photograph of a firefighter performing manual CPR in the ladder basket.

\section{Abbreviations}

CPR: Cardiopulmonary resuscitation; ECMO: Extracorporeal membrane oxygenation; eCPR: Extracorporeal cardiopulmonary resuscitation; EMS: Emergency medical services; ERC: European Resucitation Council; OHCA: Out-of-hospital cardiac arrest; ROSC: Return of spontaneous circulation

\section{Acknowledgements}

The authors would like to thank all participants for their participation and Robin Böttcher for the photographs.

\section{Authors' contributions}

All authors have contributed to study design or acquisition, analysis, and interpretation of data, have critically revised and approved the manuscript. HD and SN conceived and designed the study. HD and NA analysed the data. HD drafted the manuscript. WAW and TA contributed to the interpretation of the data and the revision of the manuscript.

\section{Funding}

No external funding was received for the conduct of this study.

\section{Availability of data and materials}

The datasets used and/or analysed during the current study are available from the corresponding author on reasonable request.

\section{Ethics approval and consent to participate}

Ethical approval was obtained from the institutional ethics committee of the Medical Faculty of the University of Cologne (approval number 17-301). All participants agreed to participate verbally.

\section{Consent for publication}

Not applicable.

\section{Competing interests}

Independent from this study, T.A received research grants from CytoSorbents, Corpuls, Medtronic, German Federal Ministry of Economics and Energy (Central innovation program for medium-sized companies, ZIM Program), and the Center of Integrated Oncology ( $\mathrm{ClO}$ ) Cologne-Bonn. The other authors report no conflicts of interest.

Received: 17 October 2019 Accepted: 5 February 2020

Published online: 04 March 2020

\section{References}

1. Michels G, Wengenmayer T, Hagl C, Dohmen C, Böttiger BW, Bauersachs J, Markewitz A, Bauer A, Gräsner JT, Pfister R, Ghanem A, Busch HJ, Kreimeier U, Beckmann A, Fischer M, Kill C, Janssens U, Kluge S, Born F, Hoffmeister HM, Preusch M, Boeken U, Riessen R, Thiele H. Recommendations for extracorporeal cardiopulmonary resuscitation (eCPR): consensus statement of DGIIN, DGK, DGTHG, DGfK, DGNI, DGAl, DIVI and GRC. Clin Res Cardiol. 2019;108:455-64.

2. Singer B, Reynolds JC, Lockey DJ, O'Brien B. Pre-hospital extra-corporeal cardiopulmonary resuscitation. Scand J Trauma Resusc Emerg Med. 2018;26:21.

3. Lamhaut L, Hutin A, Puymirat E, Jouan J, Raphalen JH, Jouffroy R, Jaffry M, Dagron C, An K, Dumas F, Marijon E, Bougouin W, Tourtier JP, Baud F, Jouven X, Danchin N, Spaulding C, Carli P. A Pre-Hospital Extracorporeal Cardio Pulmonary Resuscitation (ECPR) strategy for treatment of refractory out hospital cardiac arrest: An observational study and propensity analysis. Resuscitation. 2017:117:109-17.

4. Perkins GD, Lall R, Quinn T, Deakin CD, Cooke MW, Horton J, Lamb SE, Slowther AM, Woollard M, Carson A, Smyth M, Whitfield R, Williams A, Pocock H, Black JJ, Wright J, Han K, Gates S, PARAMEDIC trial collaborators.
Mechanical versus manual chest compression for out-of-hospital cardiac arrest (PARAMEDIC): a pragmatic, cluster randomised controlled trial. Lancet. 2015;385:947-55.

5. Wik L, Olsen JA, Persse D, Sterz F, Lozano M Jr, Brouwer MA, Westfall M, Souders CM, Malzer R, van Grunsven PM, Travis DT, Whitehead A, Herken UR, Lerner EB. Manual vs. integrated automatic load-distributing band CPR with equal survival after out of hospital cardiac arrest. The randomized CIRC trial. Resuscitation. 2014;85:741-8.

6. Rubertsson S, Lindgren E, Smekal D, Östlund O, Silfverstolpe J, Lichtveld RA, Boomars R, Ahlstedt B, Skoog G, Kastberg R, Halliwell D, Box M, Herlitz J, Karlsten R. Mechanical chest compressions and simultaneous defibrillation vs conventional cardiopulmonary resuscitation in out-of-hospital cardiac arrest: the LINC randomized trial. JAMA. 2014;311:53-61.

7. Li H, Wang D, Yu Y, Zhao X, Jing X. Mechanical versus manual chest compressions for cardiac arrest: a systematic review and meta-analysis. Scand J Trauma Resusc Emerg Med. 2016;24:10.

8. Soar J, Nolan JP, Böttiger BW, Perkins GD, Lott C, Carli P, Pellis T, Sandroni C, Skrifvars MB, Smith GB, Sunde K, Deakin CD. Adult advanced life support section Collaborators. European Resuscitation Council Guidelines for Resuscitation 2015: Section 3. Adult advanced life support. Resuscitation. 2015;95:100-47

9. Olasveengen TM, Wik L, Steen PA. Quality of cardiopulmonary resuscitation before and during transport in out-of-hospital cardiac arrest. Resuscitation. 2008;76:185-90.

10. Gässler H, Ventzke MM, Lampl L, Helm M. Transport with ongoing resuscitation: a comparison between manual and mechanical compression. Emerg Med J. 2013;30:589-92.

11. Putzer G, Braun P, Zimmermann A, Pedross F, Strapazzon G, Brugger H, Paal $P$. LUCAS compared to manual cardiopulmonary resuscitation is more effective during helicopter rescue-a prospective, randomized, cross-over manikin study. Am J Emerg Med. 2013;31:384-9.

12. Magliocca A, Olivari D, De Giorgio D, Zani D, Manfredi M, Boccardo A, Cucino A, Sala G, Babini G, Ruggeri L, Novelli D, Skrifvars MB, Hardig BM Pravettoni D, Staszewsky L, Latini R, Belloli A, Ristagno G. LUCAS Versus Manual Chest Compression During Ambulance Transport: A Hemodynamic Study in a Porcine Model of Cardiac Arrest. J Am Heart Assoc. 2019;8: e011189.

13. Gyory RA, Buchle SE, Rodgers D, Lubin JS. The Efficacy of LUCAS in Prehospital Cardiac Arrest Scenarios: A Crossover Mannequin Study. West J Emerg Med. 2017;18:437-45 doi.

14. Lyon RM, Crawford A, Crookston C, Short S, Clegg GR. The combined use of mechanical CPR and a carry sheet to maintain quality resuscitation in outof-hospital cardiac arrest patients during extrication and transport. Resuscitation. 2015:93:102-6.

15. Drennan IR, Strum RP, Byers A, Buick JE, Lin S, Cheskes S, Hu S, Morrison LJ. Rescu investigators. Out-of-hospital cardiac arrest in high-rise buildings: delays to patient care and effect on survival. CMAJ. 2016;188:413-9.

16. Lian TW, Allen JC, Ho AFW, Lim SH, Shahidah N, Ng YY, Doctor N, Leong BSH, Gan HN, Mao DR, Chia MYC, Cheah SO, Tham LP, Ong MEH. Effect of vertical location on survival outcomes for out-of-hospital cardiac arrest in Singapore. Resuscitation. 2019;139:24-32.

17. Koster RW, Beenen LF, van der Boom EB, Spijkerboer AM, Tepaske R, van der Wal AC, Beesems SG, Tijssen JG. Safety of mechanical chest compression devices AutoPulse and LUCAS in cardiac arrest: a randomized clinical trial for non-inferiority. Eur Heart J. 2017;38:3006-13.

18. Milling L, Astrup BS, Mikkelsen S. Prehospital cardiopulmonary resuscitation with manual or mechanical chest compression: A study of compressioninduced injuries. Acta Anaesthesiol Scand. 2019;63:789-95.

19. Friberg N, Schmidbauer S, Walther C, Englund E. Skeletal and soft tissue injuries after manual and mechanical chest compressions. Eur Heart J Qual Care Clin Outcomes. 2019:5:259-65.

\section{Publisher's Note}

Springer Nature remains neutral with regard to jurisdictional claims in published maps and institutional affiliations. 